\title{
Can Palm Reading Pick Up Cancer?
}

\author{
Archana Baburao ${ }^{1}$ Nandini Albur Shankaraiah² \\ ${ }^{1}$ Department of Pulmonary Medicine, Kempegowda Institute of \\ Medical Sciences, Bengaluru, Karnataka, India \\ 2Department of Dermatology, Kempegowda Institute of Medical \\ Sciences, Bengaluru, Karnataka, India
}

\section{South Asian J Cancer 2020;9:186-187.}

The association of skin with visceral cancer was first described by Hebra in $1868 .{ }^{1}$ More than 50 dermatological conditions have been reported as markers of malignancy. Paraneoplastic dermatoses are heterogeneous group of clinical manifestations that often appears benign, and they are the third most common paraneoplastic site very next to neurologic and endocrine syndromes. ${ }^{2}$ There is no relationship between the severity of symptoms and the size of the primary tumor. It commonly precedes or follows visceral cancer $^{3}$ and early recognition may result in earlier diagnosis and management of visceral cancer. At other times, the syndromes may occur late in the course of disease or may appear as the first sign of recurrence. $^{3}$

We report a 60-year-old male chronic smoker who presented with complaints of peeling of the skin all over the body sparing the face for the last 2 weeks. He also had a loss of appetite and weight that was not quantified. His past history was unremarkable. On examination, vitals were stable with a body mass index of $17.8 \mathrm{~kg} / \mathrm{m}^{2}$. He had pallor and bilateral digital clubbing. General physical examination showed prominent dermatoglyphics with exaggeration of the ridges and furrows and thickening and darkening of both palm and sole with a velvety appearance ( - Fig. 1 ). Systemic examination and routine blood investigations were normal expect for mild anemia. Skin biopsy of palm showed marked hyperkeratosis, irregular acanthosis, and mild papillomatosis ( - Fig. 2A) collectively termed as tripe palms, which can be the first sign of an undiagnosed cancer particularly gastric and lung cancer. Hence, the patient underwent a full diagnostic workup for an associated malignancy. Imaging of thorax showed necrotic lung mass with cavitation and necrotic mediastinal lymphadenopathy ( $\sim$ Fig. 3). Computed tomography-guided biopsy of lung mass was suggestive of small cell carcinoma of the lung ( - Fig. 2B). Upper gastrointestinal (GI) endoscopy and ultrasonographic abdomen, done to rule
Address for correspondence Archana Baburao, MBBS, DNB, DTCD, Department of Pulmonary Medicine, Kempegowda Institute of Medical Sciences, Bengaluru, Karnataka, India (e-mail: dr_archana82@yahoo.co.in).

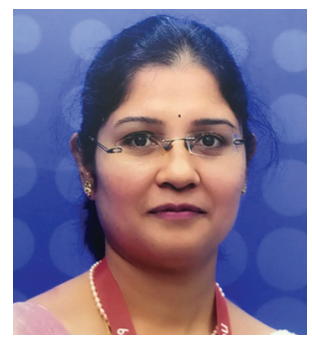

Archana Baburao, MBBS, DNB, DTCD

out GI malignancies, were normal. A diagnosis of tripe palms secondary to small cell carcinoma of the lung was made and the patient was referred to medical oncologist and started on intravenous etoposide and cisplatin in cycles. However, the patient succumbed to cancer after 1 month.

Paraneoplastic syndromes are a group of clinical disorders that are associated with malignant diseases and are not directly related to direct invasion, obstruction, or metastasis. ${ }^{3}$ Although paraneoplastic syndromes can be associated with many types of malignancies, they are most frequently associated with lung cancer occurring in $10 \%$ of these patients, ${ }^{3}$ especially small cell lung cancer (SCLC). Humoral hypercalcemia of malignancy in squamous cell carcinoma and syndrome of inappropriate antidiuretic hormone in SCLC are the two common paraneoplastic syndromes in lung cancer patients. Some of the common paraneoplastic dermatoses are listed in - Table 1. SCLC is most commonly associated with neurologic, endocrine, and rheumatic paraneoplastic syndromes. Clubbing and hypertrophic osteoarthropathy occur in 1 to $5 \%$ of all patients with SCLC and it is the most reported paraneoplastic dermatoses, ${ }^{4}$ but it is not specific to SCLC. Other paraneoplastic dermatoses reported in literature in SCLC patients include dermatomyositis, Bazex syndrome, and acquired ichthyosis.

We report a first case of SCLC that presented with tripe palms. Tripe palms consist of acanthosis palmaris, pachydermatoglyphy, and palmar hyperkeratosis where there is yellowish rugose hypertrophy of the palms, and sometimes the soles, leading to an exaggeration of the skin lines and the skin, resemble the edible lining of bovine foregut and is usually associated with paraneoplastic acanthosis nigricans. Tripe palms are usually associated with malignancy in $90 \%$ of cases, gastric and lung cancers accounting for more than $50 \%$ of cases. ${ }^{5}$ In the absence of an association with paraneoplastic acanthosis nigricans, lung cancer becomes more prevalent, 

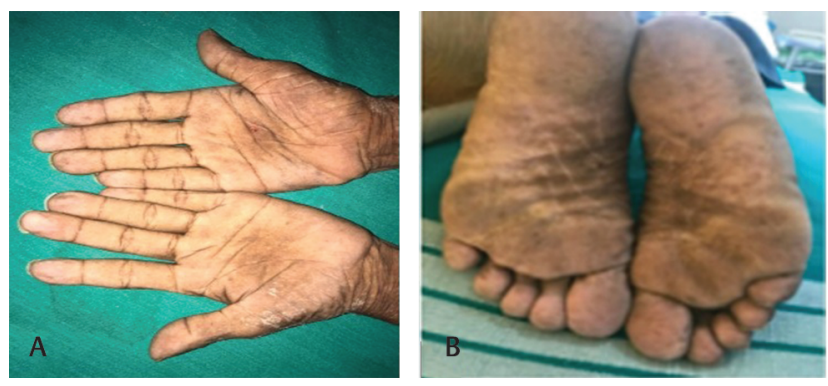

Fig. 1 Prominent dermatoglyphics with exaggeration of the ridges and furrows of palm (A) and sole (B) with a velvety appearance.
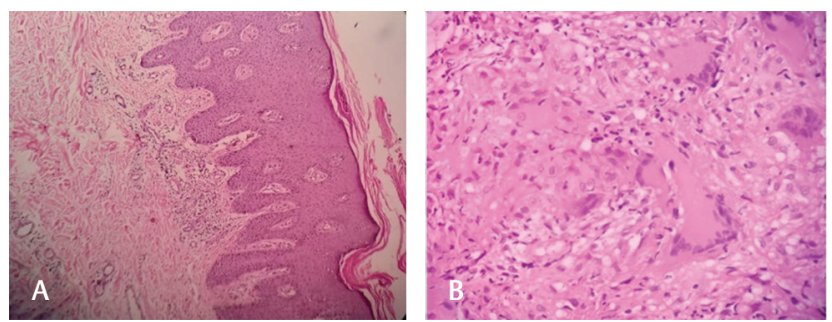

Fig. 2 (A) Skin biopsy showing epidermal hyperkeratosis, mild papillomatosis, and irregular acanthosis with unremarkable dermis. (B) Lung biopsy showing atypical cells arranged in clusters with high nucleus cytoplasm ratio, moderate amount of cytoplasm, vesicular nucleus, and prominent nucleoli.

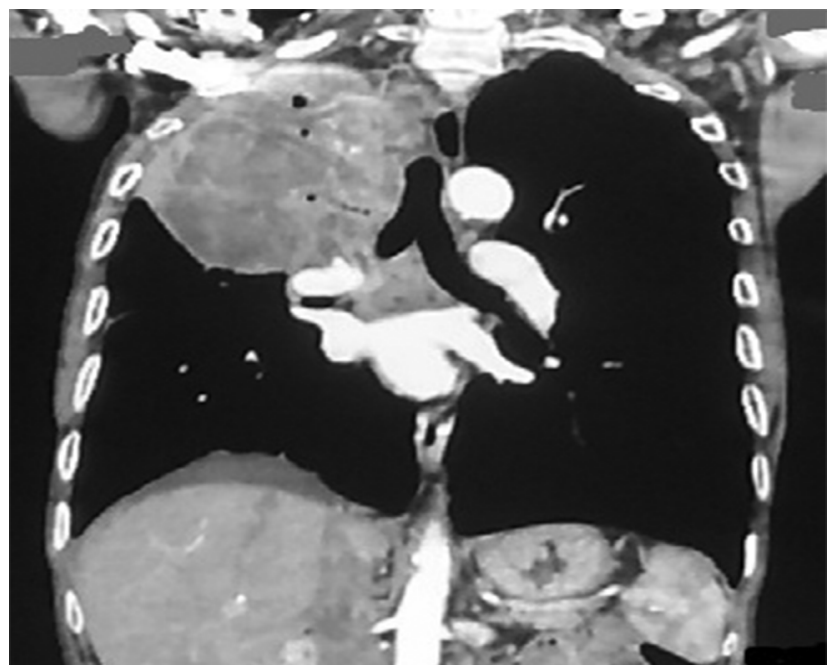

Fig. 3 Computed tomography thorax showing the right upper lobe necrotic mass with mediastinal lymph nodes.

accounting for more than $50 \%$ of cases. ${ }^{5}$ Pulmonary tumors are usually nonsmall cell, with squamous cell and adenocarcinoma described most frequently in the literature. Other neoplasms that are correlated with tripe palms include liver, gallbladder and bile duct breast, and genitourinary tract cancers.

The exact pathogenesis of acanthosis palmaris is poorly understood. Several cytokines such as transforming growth factor $\alpha$, insulin growth factor-1-like, fibroblast growth factor, and melanocyte-stimulating
Table 1 Common paraneoplastic dermatologic syndromes associated with lung cancer

\begin{tabular}{|l|}
\hline $\begin{array}{l}\text { Paraneoplastic dermatologic syndromes associated with } \\
\text { lung cancer }\end{array}$ \\
\hline Acrokeratosis paraneoplastica (Bazex syndrome) \\
\hline Acanthosis nigricans \\
\hline Tripe palms \\
\hline The sign of Leser-Trélat \\
\hline Erythema gyratum repens \\
\hline Pityriasis rubra pilaris \\
\hline Pyoderma gangrenosum \\
\hline Eosinophilic cellulitis \\
\hline Pemphigus herpetiformis \\
\hline Hypertrichosis lanuginosa acquisita \\
\hline Erythema elevatum diutinum \\
\hline Polymyositis/dermatomyositis \\
\hline Hypertrophic pulmonary osteoarthropathy \\
\hline
\end{tabular}

hormone- $\beta$ produced by neoplastic cells could be responsible for an increased proliferation of keratinocytes and the development of paraneoplastic acanthosis palmaris. ${ }^{1}$ In general, paraneoplastic dermatologic syndromes improve in response to the treatment of the underlying neoplasm; the efficacy of ectopic local treatments is partial or insufficient. Furthermore, the responsiveness to the therapy is generally weaker compared with the nonparaneoplastic equivalents.

Good understanding of paraneoplastic syndromes associated with lung cancer is necessary for their early recognition that may result in earlier diagnosis and management of lung cancer. Currently, the treatment of these syndromes consists of direct therapies aimed at the underlying lung cancer.

Funding

Nil.

Conflicts of Interest

There are no conflicts of interest.

\section{References}

1 Sneddon IB. Cutaneous manifestations of visceral malignancy. Postgrad Med J 1970;46(541):678-685

2 Kanaji N, Watanabe N, Kita N, et al. Paraneoplastic syndromes associated with lung cancer. World J Clin Oncol 2014; 5(3):197-223

3 Spiro SG, Gould MK, Colice GL; American College of Chest Physicians. Initial evaluation of the patient with lung cancer: symptoms, signs, laboratory tests, and paraneoplastic syndromes: ACCP evidenced-based clinical practice guidelines (2nd edition). Chest. 2007 Sep;132(3 Suppl):149S-160S.

4 Ito T, Goto K, Yoh K, et al. Hypertrophic pulmonary osteoarthropathy as a paraneoplastic manifestation of lung cancer. J Thorac Oncol 2010;5(7):976-980

5 Cohen PR, Grossman ME, Almeida L, Kurzrock R. Tripe palms and malignancy. J Clin Oncol 1989;7(5):669-678 